ESAIM: COCV 19 (2013) 288-300

DOI: $10.1051 / \mathrm{cocv} / 2012013$
ESAIM: Control, Optimisation and Calculus of Variations

www.esaim-cocv.org

\title{
REMARKS ON NON CONTROLLABILITY OF THE HEAT EQUATION WITH MEMORY*,**
}

\author{
Sergio Guerrero ${ }^{1}$ and Oleg Yurievich Imanuvilov ${ }^{2}$
}

\begin{abstract}
In this paper we deal with the null controllability problem for the heat equation with a memory term by means of boundary controls. For each positive final time $T$ and when the control is acting on the whole boundary, we prove that there exists a set of initial conditions such that the null controllability property fails.
\end{abstract}

Mathematics Subject Classification. 93B.

Received March 27, 2012. Revised July 26, 2011.

Published online June 12, 2012.

\section{INTRODUCTION}

Let $\Omega$ be a bounded domain with smooth boundary and let $T>0$. We consider the following control system associated to the heat equation with memory:

$$
\begin{cases}y_{t}-\Delta y-\int_{0}^{t} \Delta y \mathrm{~d} \tau=0 & \text { in } Q:=(0, T) \times \Omega, \\ y=v & \text { on }(0, T) \times \partial \Omega, \\ y(0, \cdot)=y_{0}(\cdot) & \text { in } \Omega .\end{cases}
$$

Here, $v \in L^{2}((0, T) \times \partial \Omega)$ is a control function which is acting on our system at the boundary. Furthermore, $y_{0}$ is the initial condition which is supposed to be in $L^{2}(\Omega)$. Under these assumptions, it is classical to see (e.g. by transposition method [6]) that there exists a unique solution $y$ of system (1.1) which belongs to the space $L^{2}(Q)$ and a constant $K_{0}>0$ such that

$$
\|y\|_{L^{2}(Q)} \leq K_{0}\left(\left\|y_{0}\right\|_{L^{2}(\Omega)}+\|v\|_{L^{2}((0, T) \times \partial \Omega)}\right) .
$$

The null controllability property for system (1.1) reads as follows: given $y_{0} \in L^{2}(\Omega)$, does there exist a control $v \in L^{2}((0, T) \times \partial \Omega)$ such that the corresponding solution of $(1.1)$ satisfies $y(T, \cdot) \equiv 0$ in $L^{2}(\Omega)$ ? This property

Keywords and phrases. Controllability, heat equation with memory.

* SG was partially supported by the "Agence Nationale de la Recherche" (ANR), Project CISIFS, grant ANR-09-BLAN-0213-02.

** This work is partially supported by NSF Grant DMS 0808130.

1 Université Pierre et Marie Curie - Paris 6, UMR 7598 Laboratoire Jacques-Louis Lions, 75005 Paris, France. guerrero@ann.jussieu.fr.

2 Department of Mathematics, Colorado State University, 101 Weber Building, Fort Collins, 80523-1874 CO, USA.

oleg@math. colostate.edu. 
is very well-known to be true for the heat equation (see, for instance, $[1,2,4,7]$ ). The purpose of this paper is to prove that this is not the case of the heat equation with a memory term.

The problem (1.1) naturally appears in some models developed for the approximation of the Navier-Stokes system (see, for instance, [8]).

In [3], for a one-dimensional heat equation with memory, the authors proved the lack of "controllability to the rest" for some initial conditions when controlling through one endpoint. This notion of controllability means that, with the help of a control, one can prove that $y(T, \cdot) \equiv 0$ and $\int_{0}^{T} y(t, \cdot) \mathrm{d} t \equiv 0$.

As we said above, the main result of this paper states that the null controllability of system (1.1) does not hold for all initial conditions. The precise formulation of this result is given in the following theorem:

Theorem 1.1. Let $T>0$. Then, there exist initial conditions $y_{0} \in L^{2}(\Omega)$ such that for any control function $v \in L^{2}((0, T) \times \partial \Omega)$ the associated solution $y \in L^{2}(Q)$ to $(1.1)$ is not identically equal zero at time $T$.

In order to prove Theorem 1.1, we will suppose that $\Omega$ is a cube. Then, if $\Omega$ is a general bounded domain in $\mathbf{R}^{N}$ we consider a cube $K \subset \Omega$. Once the proof is established for $K$, we would have that for any fixed positive $T$ there exists an initial condition $\hat{y}_{0} \in L^{2}(K)$ such that for any boundary control $v \in L^{2}((0, T) \times \partial K)$ the solution $y$ at moment $T$ is not identically equal zero. We extend $\hat{y}_{0}$ on $\Omega \backslash K$. Obviously for such an initial condition the null controllability property at moment $T$ fails.

Consider now the similar problem

$$
\begin{cases}y_{t}-\Delta y+\int_{0}^{t} y \mathrm{~d} \tau=0 & \text { in } Q \\ y=v & \text { on }(0, T) \times \partial \Omega, \\ y(0, \cdot)=y_{0}(\cdot) & \text { in } \Omega .\end{cases}
$$

Theorem 1.2. Let $T>0$. Then, there exist initial conditions $y_{0} \in L^{2}(\Omega)$ such that for any control function $v \in L^{2}((0, T) \times \partial \Omega)$ the associated solution $y \in L^{2}(Q)$ to $(1.3)$ is not identically equal to zero at time $T$.

The proof of this theorem is completely analogous to that of Theorem 1.1, so we omit it.

Remark 1.3. The same non null controllability results hold for interior controls $v 1_{\omega}$, where $\omega$ is an open set satisfying $\bar{\omega} \subset \Omega$.

Remark 1.4. There have recently been several published papers which claim to prove the observability inequality for the heat equation with memory. In the process of the proof of Theorem 1.1 we establish in particular that this observability inequality is not true.

Since the proof of Theorem 1.1 is rather technical, we have decided to first provide the proof for the onedimensional case. This is done in Section 1. Finally, in the second section we prove Theorem 1.1 in any dimension.

\section{ONE DIMENSIONAL CASE}

In this section, we prove the following result:

Theorem 2.1. Let $T>0$. Then, there exist initial conditions $\bar{y}_{0} \in L^{2}(0,1)$ such that, for any control functions $v_{1}, v_{2} \in L^{2}(0, T)$, the associated solution $y \in L^{2}(Q)$ to

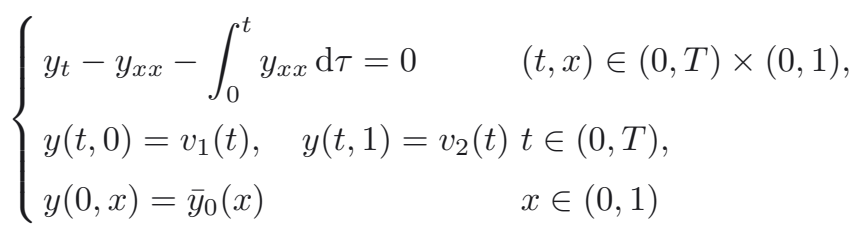


satisfies

$$
y(T, \cdot) \not \equiv 0 \text { in }(0,1)
$$

Proof. Let us introduce the adjoint system associated to our control problem (2.1):

$$
\begin{cases}-\varphi_{t}-\varphi_{x x}-\int_{t}^{T} \varphi_{x x} \mathrm{~d} \tau=0 & (t, x) \in(0, T) \times(0,1), \\ \varphi(t, 0)=0, \quad \varphi(t, 1)=0 & t \in(0, T), \\ \varphi(T, x)=\varphi_{T}(x) & x \in(0,1),\end{cases}
$$

for $\varphi_{T} \in L^{2}(0,1)$. It is very well-known by now (see, for instance, [7] or [5]) that the null controllability of system (2.1) with $L^{2}$-controls depending continuously on $y_{0}$ is equivalent to the following observability inequality:

$$
\int_{0}^{1}|\varphi(0, x)|^{2} \mathrm{~d} x \leq C_{o b s} \int_{0}^{T}\left(\left|\varphi_{x}(t, 0)\right|^{2}+\left|\varphi_{x}(t, 1)\right|^{2}\right) \mathrm{d} t .
$$

Our goal in this proof is to construct, for all sufficiently large $M$, a sequence of solutions $\left\{\varphi^{M}\right\}$ of $(2.3)$ such that $\left\|\left.\varphi^{M}\right|_{t=0}\right\|_{L^{2}(0,1)}$ is estimated from below by $C / M^{2}$ for some $C$ independent of $M$ and the quantity

$$
\left(\left\|\varphi_{x \mid x=0}^{M}\right\|_{L^{2}(0, T)}^{2}+\left\|\varphi_{x \mid x=1}^{M}\right\|_{L^{2}(0, T)}^{2}\right)^{1 / 2}
$$

is estimated from above by $C / M^{3}$ for some (maybe different) $C>0$ independent of $M$. Then, based on the properties of this sequence, we will construct an initial condition $\bar{y}_{0} \in L^{2}(0,1)$ such that system $(2.1)$ is not null controllable.

First, let

$$
w_{j}(x):=\sin (j \pi x) \quad x \in(0,1), \lambda_{j}=(j \pi)^{2}, \forall j \in \mathbf{N}_{*}:=\mathbf{N} \backslash\{0\}
$$

be the eigenfunctions and eigenvalues of the Laplace operator with the Dirichlet boundary conditions in $(0,1)$ :

$$
\left\{\begin{array}{l}
-\Delta w_{j}=\lambda_{j} w_{j} \text { in }(0,1), \\
w_{j}(0)=w_{j}(1)=0
\end{array}\right.
$$

Let us write

$$
\varphi_{T}(x)=\sum_{j \geq 1} \beta_{j} w_{j}(x) \quad \forall x \in(0,1)
$$

for a sequence $\left\{\beta_{j}\right\}_{j \geq 1} \in \ell^{2}:=\left\{\left\{y_{j}\right\} \in \mathbf{R}^{\mathbf{N}}: \sum_{j \in \mathbf{N}_{*}}\left|y_{j}\right|^{2}<+\infty\right\}$.

The solution $\varphi$ of $(2.3)$ can be written as follows:

$$
\varphi(t, x)=\sum_{j \geq 1} \alpha_{j}(t) w_{j}(x) \quad(t, x) \in(0, T) \times(0,1),
$$

where the function $\alpha_{j}(t)$ satisfies

$$
\begin{cases}-\alpha_{j}^{\prime \prime}+\lambda_{j} \alpha_{j}^{\prime}-\lambda_{j} \alpha_{j}=0 & \text { in }(0, T), \\ \alpha_{j}(T)=\beta_{j},-\alpha_{j}^{\prime}(T)+\lambda_{j} \alpha_{j}(T)=0 . & \end{cases}
$$

That is to say,

$$
\alpha_{j}(t)=C_{1, j} \mathrm{e}^{\mu_{j}^{+}(T-t)}+C_{2, j} \mathrm{e}^{\mu_{j}^{-}(T-t)} \quad t \in(0, T),
$$


with

$$
\mu_{j}^{ \pm}=-\frac{\lambda_{j}}{2} \mp \frac{\sqrt{\lambda_{j}^{2}-4 \lambda_{j}}}{2}=\frac{\lambda_{j}}{2}\left(-1 \mp \sqrt{1-4 / \lambda_{j}}\right)
$$

and

$$
C_{1, j}=\beta_{j} \frac{\mu_{j}^{-}+\lambda_{j}}{\mu_{j}^{-}-\mu_{j}^{+}}=\beta_{j} \frac{1+\sqrt{1-4 / \lambda_{j}}}{2 \sqrt{1-4 / \lambda_{j}}}, C_{2, j}=\beta_{j} \frac{\mu_{j}^{+}+\lambda_{j}}{\mu_{j}^{+}-\mu_{j}^{-}}=\beta_{j} \frac{-1+\sqrt{1-4 / \lambda_{j}}}{2 \sqrt{1-4 / \lambda_{j}}} .
$$

Observe that $\mu_{j}^{+}$gives faith of the parabolic character of this equation since it tends to $-\infty$, while $\mu_{j}^{-}$represents a completely different type of behavior $\left(\mu_{j}^{-} \rightarrow-1\right.$ when $\left.j \rightarrow+\infty\right)$.

With this notation, the integral

$$
\int_{0}^{1}|\varphi(0, x)|^{2} \mathrm{~d} x
$$

is given by

$$
\int_{0}^{1}\left|\sum_{j \geq 1}\left(C_{1, j} \mathrm{e}^{\mu_{j}^{+} T}+C_{2, j} \mathrm{e}^{\mu_{j}^{-} T}\right) w_{j}(x)\right|^{2} \mathrm{~d} x=\frac{1}{2} \sum_{j \geq 1}\left(C_{1, j} \mathrm{e}^{\mu_{j}^{+} T}+C_{2, j} \mathrm{e}^{\mu_{j}^{-} T}\right)^{2}
$$

thanks to the orthogonality of the eigenfunctions in $L^{2}(0,1)$ and the fact that $\left\|w_{j}\right\|_{L^{2}(0,1)}^{2}=1 / 2$.

On the other hand, the term

$$
\int_{0}^{T} \mathrm{e}^{2(T-t)}\left(\left|\varphi_{x}(t, 0)\right|^{2}+\left|\varphi_{x}(t, 1)\right|^{2}\right) \mathrm{d} t
$$

is given by

$$
\begin{aligned}
\pi^{2} \int_{0}^{T}\left(g \sum_{j \geq 1} j\left(C_{1, j} \mathrm{e}^{\left(1+\mu_{j}^{+}\right)(T-t)}+C_{2, j} \mathrm{e}^{\left(1+\mu_{j}^{-}\right)(T-t)}\right)\right)^{2} \mathrm{~d} t \\
+\pi^{2} \int_{0}^{T}\left(\sum_{j \geq 1}(-1)^{j} j\left(C_{1, j} \mathrm{e}^{\left(1+\mu_{j}^{+}\right)(T-t)}+C_{2, j} \mathrm{e}^{\left(1+\mu_{j}^{-}\right)(T-t)}\right)\right)^{2} \mathrm{~d} t .
\end{aligned}
$$

Of course, it is equivalent to estimate (2.8) or $\left\|\varphi_{x \mid x=0}\right\|_{L^{2}(0, T)}^{2}+\left\|\varphi_{x \mid x=1}\right\|_{L^{2}(0, T)}^{2}$ but we have chosen to estimate (2.8) since the term $\mathrm{e}^{2(T-t)}$ will somehow simplify the computations.

The idea of the proof is to find a particular choice of $\left\{\beta_{j}\right\}$ so that the ratio between (2.7) and (2.8) is large enough. Let's make this choice so that just a finite number of $\beta_{j}$ 's will be different from zero. In fact, let $M$ be a large entire parameter; we take

$$
\beta_{j}=0 \text { for all } j \notin\{12 M+2 k: k \in \mathbf{N}, 1 \leq k \leq 6\} .
$$




\section{Estimate from above of (2.8)}

A direct computation shows that we can estimate (2.8) by $A:=A_{1}+A_{2}$, where

$$
A_{1}:=4 \pi^{2} \int_{0}^{T}\left(\sum_{\substack{j=12 M+2 k, k=1}}^{6} j C_{1, j} \mathrm{e}^{\left(1+\mu_{j}^{+}\right)(T-t)}\right)^{2} \mathrm{~d} t
$$

and

$$
A_{2}:=4 \pi^{2} \int_{0}^{T}\left(\sum_{\substack{j=12 M+2 k, k=1}}^{6} j C_{2, j} \mathrm{e}^{\left(1+\mu_{j}^{-}\right)(T-t)}\right)^{2} \mathrm{~d} t .
$$

We give an estimate of the first and of the second term separately:

- Estimate of $A_{1}$. Taking into account the expression of the eigenvalues and using the notation

$$
(k-3 / 2) !=(k-3 / 2)(k-5 / 2) \ldots 1 / 2 \quad \forall k \geq 2 \quad \text { and } \quad(-1 / 2) !=1,
$$

we find

$$
\begin{aligned}
\exp \left\{\left(1+\mu_{j}^{+}\right)(T-t)\right\} & =\exp \left\{-\lambda_{j}(T-t)\right\} \exp \left\{(T-t)\left(1+\sum_{k \geq 1} \frac{(k-3 / 2) !}{k !}\left(\frac{4}{\lambda_{j}}\right)^{k-1}\right)\right\} \\
& :=\exp \left\{\left(2-\lambda_{j}\right)(T-t)\right\} \exp \left\{(T-t) B_{j}\right\}
\end{aligned}
$$

where $B_{j}$ goes to zero (like $j^{-2}$ ) as $j$ goes to infinity.

We can rewrite $A_{1}$ as follows:

$$
A_{1}=4 \pi^{2} \int_{0}^{T} \mathrm{e}^{\left(4-288 M^{2} \pi^{2}\right)(T-t)} g_{M}(t) \mathrm{d} t
$$

where $g_{M}(t)=f_{M}(t)^{2}, \forall t \in[0, T]$ and $f_{M}$ is defined as follows:

$$
f_{M}(t)=\sum_{k=1}^{6}(12 M+2 k) C_{1,12 M+2 k} \exp \left\{\left(-\left(48 k M+4 k^{2}\right) \pi^{2}+B_{12 M+2 k}\right)(T-t)\right\} .
$$

Let us now impose the following seven conditions:

$$
g_{M}(T)=g_{M}^{\prime}(T)=g_{M}^{\prime \prime}(T)=g_{M}^{\prime \prime \prime}(T)=g_{M}^{I V}(T)=g_{M}^{V}(T)=g_{M}^{V I}(T)=0 .
$$

These conditions are fulfilled just by imposing four linear equations (which correspond to $f_{M}(T)=f_{M}^{\prime}(T)=$ $\left.f_{M}^{\prime \prime}(T)=f_{M}^{\prime \prime \prime}(T)=0\right)$ :

$$
\left\{\begin{array}{l}
\sum_{k=1}^{6}(12 M+2 k) C_{1,12 M+2 k}\left(\left(48 k M+4 k^{2}\right) \pi^{2}-B_{12 M+2 k}\right)^{\ell}=0, \\
\text { for every } \ell=0,1,2,3 .
\end{array}\right.
$$

Here we have four linear equations and six unknowns $\left\{C_{1,12 M+2 k}\right\}_{k=1}^{6}$. Hence the set of nontrivial solutions of (2.15) is nonempty. Moreover, since (2.15) is a linear homogeneous system, we can choose a nontrivial solution $\left\{C_{1,12+2 k}\right\}_{k=1}^{6}$ which is bounded independently of $M$. 
Then, from (2.14) and using some integrations by parts, we have that

$$
\int_{0}^{T} \mathrm{e}^{\left(4-288 M^{2} \pi^{2}\right)(T-t)} g_{M}(t) \mathrm{d} t=\sum_{\ell=0}^{6} \frac{\mathrm{e}^{\left(4-288 M^{2} \pi^{2}\right) T}}{\left(4-288 M^{2} \pi^{2}\right)^{\ell+1}} g_{M}^{(\ell)}(0)-\int_{0}^{T} \frac{\mathrm{e}^{\left(4-288 M^{2} \pi^{2}\right)(T-t)}}{\left(4-288 M^{2} \pi^{2}\right)^{7}} g_{M}^{(6)}(t) \mathrm{d} t .
$$

Observe that the $\ell$ th derivative of the function $g_{M}$ is estimated by $M^{2+\ell}$ for all $\ell \in \mathbf{N}$. The conclusion is that the term $(2.12)$ is estimated by $1 / M^{6}$.

- Estimate of $A_{2}$. We develop in power series for $j$ large enough:

$$
C_{2, j}=\frac{\beta_{j}}{2}\left(1-\left(1-4 / \lambda_{j}\right)^{-1 / 2}\right)=-\frac{\beta_{j}}{2} \sum_{k \geq 1} \frac{(k-1 / 2) !}{k !}\left(\frac{4}{\lambda_{j}}\right)^{k},
$$

and

$$
\mathrm{e}^{\left(1+\mu_{j}^{-}\right)(T-t)}=\exp \left\{\left(1-\frac{\lambda_{j}}{2}+\frac{\lambda_{j}}{2}\left(1-\sum_{k \geq 1} \frac{(k-3 / 2) !}{2(k !)}\left(\frac{4}{\lambda_{j}}\right)^{k}\right)\right)(T-t)\right\} .
$$

Observe that, since $j$ is large enough we can suppose in particular that

$$
\frac{T-t}{(\pi j)^{2}}<1 \quad \forall t \in[0, T]
$$

and so we obtain

$$
\mathrm{e}^{\left(1+\mu_{j}^{-}\right)(T-t)}=1-\frac{T-t}{\lambda_{j}}+O\left(\lambda_{j}^{-2}\right) .
$$

From the previous expressions (2.16) and (2.17) and the fact that $\lambda_{j}=(\pi j)^{2}$, the $j$ th term in the expression of $A_{2}$ (see (2.10)) is given by

We impose that

$$
-j \beta_{j}\left(\frac{1}{\pi^{2} j^{2}}+\frac{3-(T-t)}{\pi^{4} j^{4}}+O\left(\lambda_{j}^{-3}\right)\right) .
$$

$$
\sum_{k=1}^{6} \frac{\beta_{12 M+2 k}}{12 M+2 k}=0
$$

Thanks to (2.6), this equation reads

$$
\sum_{k=1}^{6} \frac{C_{1,12 M+2 k} \sqrt{1-4 / \lambda_{12 M+2 k}}}{(12 M+2 k)\left(1+\sqrt{1-4 / \lambda_{12 M+2 k}}\right)}=0,
$$

where we recall that $\lambda_{j}=(\pi j)^{2}$. Together with equations (2.15), this gives a linear homogeneous system of five equations with six unknowns. Since we have supposed that $\left\{C_{1,12 M+2 k}\right\}_{k=1}^{6}$ are bounded independently of $M$, it is easy to see from the expression of $C_{1, j}$ (see (2.6)) that $\left\{\beta_{12 M+2 k}\right\}_{k=1}^{6}$ are also bounded independently of $M$. Consequently, taking into account the definition of $A_{2}$ (see (2.10)) and (2.18), we have

$$
A_{2}=2 \int_{0}^{T}\left(\sum_{\substack{j=12 M+2 k, k=1}}^{6} j \beta_{j}\left(\frac{1}{\pi^{2} j^{2}}+\frac{3-(T-t)}{\pi^{4} j^{4}}+O\left(\lambda_{j}^{-3}\right)\right)\right)^{2} \mathrm{~d} t .
$$

Then, using (2.19), we get

$$
A_{2}=2 \int_{0}^{T}\left(\sum_{\substack{j=12 M+2 k, k=1}}^{6} j \beta_{j}\left(\frac{3-(T-t)}{\pi^{4} j^{4}}+O\left(\lambda_{j}^{-3}\right)\right)\right)^{2} \mathrm{~d} t,
$$

which implies that $A_{2}$ is estimated by $1 / M^{6}$. 


\section{Estimate from below of (2.7)}

One can estimate from below the term (2.7) as follows:

$$
\begin{aligned}
I & =\frac{1}{2} \sum_{j \geq 1}\left(C_{1, j} \mathrm{e}^{\mu_{j}^{+} T}+C_{2, j} \mathrm{e}^{\mu_{j}^{-} T}\right)^{2}=\frac{1}{2} \sum_{k=1}^{6}\left(C_{1,12 M+2 k} \mathrm{e}^{\mu_{12 M+2 k}^{+} T}+C_{2,12 M+2 k} \mathrm{e}^{\mu_{12 M+2 k}^{-} T}\right)^{2} \\
& \geq \frac{1}{2} \sum_{k=1}^{6}\left(-3 C_{1,12 M+2 k}^{2} \mathrm{e}^{2 \mu_{12 M+2 k}^{+} T}+\frac{3 C_{2,12 M+2 k}^{2}}{4} \mathrm{e}^{2 \mu_{12 M+2 k}^{-} T}\right) \\
& =\frac{1}{2} \sum_{k=1}^{6}\left(-3 C_{1,12 M+2 k}^{2} \mathrm{e}^{2 \mu_{12 M+2 k}^{+} T}+\frac{3 \beta_{12 M+2 k}^{2}}{4}\left(\frac{\mu_{12 M+2 k}^{+}+\lambda_{12 M+2 k}}{\mu_{12 M+2 k}^{+}-\mu_{12 M+2 k}^{-}}\right)^{2} \mathrm{e}^{2 \mu_{12 M+2 k}^{-} T}\right) .
\end{aligned}
$$

Observe that, from (2.16) and the fact that $\mu_{j}^{-} \rightarrow-1$ as $j \rightarrow+\infty$, we have that

$$
\inf _{1 \leq k \leq 6}\left(\frac{\mu_{12 M+2 k}^{+}+\lambda_{12 M+2 k}}{\mu_{12 M+2 k}^{+}-\mu_{12 M+2 k}^{-}}\right)^{2} \geq \frac{C}{M^{4}},
$$

for some $C>0$ independent of $M$, while $C_{1,12 M+2 k}^{2} \mathrm{e}^{2 \mu_{12 M+2 k}^{+} T}$ can be estimated by $\widehat{C} \mathrm{e}^{-M^{2} T}$. Consequently,

$$
I \geq \frac{\widetilde{C}}{M^{4}}
$$

with $\widetilde{C}>0$ independent of $M$. That is to say, we have

$$
\left\|\varphi^{M}(0, \cdot)\right\|_{L^{2}(0,1)}^{2} \geq \frac{C_{0}}{M^{4}}
$$

where $C_{0}>0$ is independent of $M$.

\section{Construction of the initial condition}

Finally, we construct an initial condition $\bar{y}_{0} \in L^{2}(0,1)$ such that the null controllability of (1.1) does not hold. In fact, from (2.7) and (2.21) we deduce the existence of $k_{0} \in\{1,2,3,4,6\}$ such that

$$
\frac{1}{2}\left(C_{1,12 M+2 k_{0}} \mathrm{e}^{\mu_{12 M+2 k_{0}}^{+} T}+C_{2,12 M+2 k_{0}} \mathrm{e}^{\mu_{12 M+2 k_{0}}^{-} T}\right)^{2} \geq \frac{C_{0}}{6 M^{4}} .
$$

Then, we define

$$
\bar{y}_{0}=\sum_{\ell \geq 1} \frac{1}{\ell^{3 / 4}} w_{12 \ell+2 k_{0}} \in L^{2}(0,1)
$$

(recall that the eigenfunction $w_{j}$ was defined in (2.4)). Let us prove that for any $v_{1}, v_{2} \in L^{2}(0, T)$, the solution $y$ of

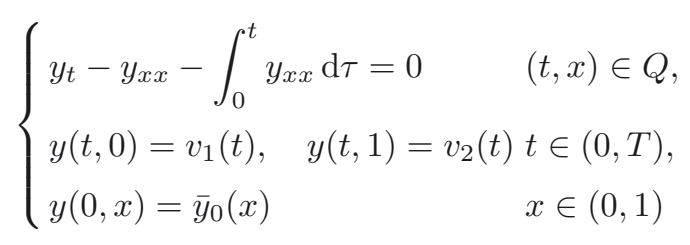


satisfies $y(T, \cdot) \not \equiv 0$. Arguing by contradiction, let $v_{1}, v_{2} \in L^{2}(0, T)$ be such that $y(T, x)=0$ for all $x \in(0,1)$. Then, by duality with $\varphi^{M}$, we have

$$
\begin{aligned}
\int_{0}^{1} \varphi^{M}(0, x) \bar{y}_{0}(x) \mathrm{d} x & +\int_{0}^{T} v_{1}(t) \varphi_{x}^{M}(t, 0) \mathrm{d} x+\int_{0}^{T}\left(\int_{0}^{t} v_{1}(s) \mathrm{d} s\right) \varphi_{x}^{M}(t, 0) \mathrm{d} t \\
& -\int_{0}^{T} v_{2}(t) \varphi_{x}^{M}(t, 1) \mathrm{d} x+\int_{0}^{T}\left(\int_{0}^{t} v_{2}(s) \mathrm{d} s\right) \varphi_{x}^{M}(t, 1) \mathrm{d} t=0 .
\end{aligned}
$$

From the previous choice of $\bar{y}_{0}$ and $(2.22)$, we have that

$$
\left|\int_{0}^{1} \varphi^{M}(0, x) \bar{y}_{0}(x) \mathrm{d} x\right| \geq \frac{C_{1}}{M^{11 / 4}} .
$$

Finally, since the term in $(2.8)$ is estimated by $C / M^{6}$, we have that

$$
\begin{aligned}
\mid \int_{0}^{T} v_{1}(t) \varphi_{x}^{M}(t, 0) \mathrm{d} x & +\int_{0}^{T}\left(\int_{0}^{t} v_{1}(s) \mathrm{d} s\right) \varphi_{x}^{M}(t, 0) \mathrm{d} t \\
& -\int_{0}^{T} v_{2}(t) \varphi_{x}^{M}(t, 1) \mathrm{d} x+\int_{0}^{T}\left(\int_{0}^{t} v_{2}(s) \mathrm{d} s\right) \varphi_{x}^{M}(t, 1) \mathrm{d} t \mid \leq \frac{C}{M^{3}} .
\end{aligned}
$$

This contradicts identity (2.24) by taking $M$ large enough.

This ends the proof of Theorem 2.1 in dimension 1.

\section{N-DIMENSIONAL CASE}

As explained in the introduction, it suffices to prove the desired result when $\Omega$ is a cube:

$$
\Omega:=\left\{\left(x_{1}, \ldots, x_{N}\right) \in \mathbf{R}^{N}: 0<x_{k}<1,1 \leq k \leq N\right\} .
$$

Let us introduce the adjoint system associated to our control problem (1.1):

$$
\begin{cases}-\varphi_{t}-\Delta \varphi-\int_{t}^{T} \Delta \varphi \mathrm{d} \tau=0 & \text { in }(0, T) \times \Omega, \\ \varphi=0 & \text { on }(0, T) \times \partial \Omega, \\ \varphi(T, \cdot)=\varphi_{T}(\cdot) & \text { in } \Omega,\end{cases}
$$

for $\varphi_{T} \in L^{2}(\Omega)$.

Similarly to the 1 -D case, let

$$
w_{\boldsymbol{j}}(x):=\prod_{k=1}^{N} \sin \left(j_{k} \pi x_{k}\right) x_{k} \in(0,1), \lambda_{\boldsymbol{j}}=\pi^{2} \sum_{k=1}^{N} j_{k}^{2}=\pi^{2}|\boldsymbol{j}|^{2}, \forall \boldsymbol{j}=\left(j_{1}, \ldots, j_{N}\right) \in \mathbf{N}_{*}^{N}
$$

be the eigenfunctions and eigenvalues of the Laplace operator with zero Dirichlet boundary conditions in $\Omega$ :

$$
\begin{cases}-\Delta w_{j}=\lambda_{j} w_{j} & \text { in } \Omega \\ w_{j}=0 & \text { on } \partial \Omega .\end{cases}
$$

Let us write

$$
\varphi_{T}(x)=\sum_{j \in \mathbf{N}_{*}^{N}} \beta_{\boldsymbol{j}} w_{\boldsymbol{j}}(x) \quad \forall x \in \Omega
$$

for a sequence $\left\{\beta_{\boldsymbol{j}}\right\}_{\boldsymbol{j} \in \mathbf{N}_{*}^{N}} \in \ell^{2}$. 
The solution $\varphi$ of (3.1) can be written as follows:

$$
\varphi(t, x)=\sum_{\boldsymbol{j} \in \mathbf{N}_{*}^{N}} \alpha_{\boldsymbol{j}}(t) w_{\boldsymbol{j}}(x) \quad(t, x) \in(0, T) \times \Omega,
$$

where $\alpha_{j}$ satisfies

$$
\begin{cases}-\alpha_{\boldsymbol{j}}^{\prime \prime}+\lambda_{\boldsymbol{j}} \alpha_{\boldsymbol{j}}^{\prime}-\lambda_{\boldsymbol{j}} \alpha_{\boldsymbol{j}}=0 & \text { in }(0, T), \\ \alpha_{\boldsymbol{j}}(T)=\beta_{\boldsymbol{j}},-\alpha_{\boldsymbol{j}}^{\prime}(T)+\lambda_{\boldsymbol{j}} \alpha_{\boldsymbol{j}}(T)=0 & \end{cases}
$$

That is to say,

$$
\alpha_{\boldsymbol{j}}(t)=C_{1, \boldsymbol{j}} \mathrm{e}^{\mu_{\boldsymbol{j}}^{+}(T-t)}+C_{2, \boldsymbol{j}} \mathrm{e}^{\mu_{\boldsymbol{j}}^{-}(T-t)} \quad t \in(0, T)
$$

with

$$
\mu_{j}^{ \pm}=-\frac{\lambda_{j}}{2} \mp \frac{\sqrt{\lambda_{j}^{2}-4 \lambda_{j}}}{2}=\frac{\lambda_{j}}{2}\left(-1 \mp \sqrt{1-4 / \lambda_{j}}\right)
$$

and

$$
C_{1, j}=\beta_{j} \frac{\mu_{j}^{-}+\lambda_{j}}{\mu_{j}^{-}-\mu_{j}^{+}}=\beta_{j} \frac{1+\sqrt{1-4 / \lambda_{j}}}{2 \sqrt{1-4 / \lambda_{j}}}, C_{2, j}=\beta_{j} \frac{\mu_{j}^{+}+\lambda_{j}}{\mu_{j}^{+}-\mu_{j}^{-}}=\beta_{j} \frac{-1+\sqrt{1-4 / \lambda_{j}}}{2 \sqrt{1-4 / \lambda_{j}}} .
$$

Again, $\mu_{j}^{+}$goes to $-\infty$ as $|\boldsymbol{j}| \rightarrow+\infty$, while $\mu_{\boldsymbol{j}}^{-}$tends to -1 when $|\boldsymbol{j}| \rightarrow+\infty$.

With this notation, the quantity $\|\varphi(0, \cdot)\|_{L^{2}(\Omega)}^{2}$ is given by

$$
\int_{\Omega}\left|\sum_{\boldsymbol{j} \in \mathbf{N}_{*}^{N}}\left(C_{1, j} \mathrm{e}^{\mu_{j}^{+} T}+C_{2, \boldsymbol{j}} \mathrm{e}^{\mu_{j}^{-} T}\right) w_{\boldsymbol{j}}(x)\right|^{2} \mathrm{~d} x=\frac{1}{2^{N}} \sum_{\boldsymbol{j} \in \mathbf{N}_{*}^{N}}\left(C_{1, j} \mathrm{e}^{\mu_{j}^{+} T}+C_{2, \boldsymbol{j}} \mathrm{e}^{\mu_{j}^{-} T}\right)^{2},
$$

thanks to the orthogonality of the eigenfunctions in $L^{2}(\Omega)$ and the identity $\left\|w_{\boldsymbol{j}}\right\|_{L^{2}(\Omega)}^{2}=1 / 2^{N}$ for all $\boldsymbol{j} \in \mathbf{N}_{*}^{N}$.

Consider the quantity

$$
\left\|\mathrm{e}^{T-t} \frac{\partial \varphi}{\partial n}\right\|_{L^{2}((0, T) \times \partial \Omega)}^{2}
$$

Using (2.5), this norm is given by

$$
\begin{aligned}
& \frac{\pi^{2}}{2^{N-1}} \int_{0}^{T} \sum_{i=1}^{N} \sum_{\substack{k=1 \\
k \neq i}}^{\infty}\left(\sum_{j_{i}=1}^{\infty} j_{i}\left(C_{1, j} \mathrm{e}^{\left(1+\mu_{j}^{+}\right)(T-t)}+C_{2, j} \mathrm{e}^{\left(1+\mu_{j}^{-}\right)(T-t)}\right)\right)^{2} \mathrm{~d} t \\
& +\frac{\pi^{2}}{2^{N-1}} \int_{0}^{T} \sum_{i=1}^{N} \sum_{\substack{j_{k}=1 \\
k \neq i}}^{\infty}\left(\sum_{j_{i}=1}^{\infty} j_{i}(-1)^{j_{i}}\left(C_{1, j} \mathrm{e}^{\left(1+\mu_{j}^{+}\right)(T-t)}+C_{2, j} \mathrm{e}^{\left(1+\mu_{j}^{-}\right)(T-t)}\right)\right)^{2} \mathrm{~d} t .
\end{aligned}
$$

Here, we have used that for any $i \in\{1, \ldots, N\}$ and any $\boldsymbol{h}, \boldsymbol{\ell} \in \mathbf{N}_{*}^{N-1}$ we have

$$
\int_{x_{i}=0} \prod_{\substack{k=1 \\ k \neq i}}^{N} \prod_{\substack{m=1 \\ m \neq i}}^{N} \sin \left(h_{k} \pi x_{k}\right) \sin \left(\ell_{m} \pi x_{m}\right) \mathrm{d} x^{\prime}= \begin{cases}1 / 2^{N-1} & \text { if } \boldsymbol{h}=\boldsymbol{\ell}, \\ 0 & \text { otherwise }\end{cases}
$$

and $\left.\cos \left(j_{i} \pi x_{i}\right)\right|_{x_{i}=1}=(-1)^{j_{i}}$. 
Now, we choose only a finite number of $C_{1, j}$ 's and $C_{2, j}$ 's to be non-zero, so that the previous expression can be estimated by

$$
\frac{\pi^{2}}{2^{N-2}}\left(L_{1}+L_{2}+L_{3}+L_{4}\right)
$$

where the terms $L_{i}(1 \leq i \leq 4)$ are given by

$$
L_{1}=\int_{0}^{T} \sum_{i=1}^{N} \sum_{j_{i}^{\prime} \in \mathbb{N}_{*}^{N-1}}\left(\sum_{j_{i}=1}^{\infty} j_{i} C_{1, j} \mathrm{e}^{\left(1+\mu_{j}^{+}\right)(T-t)}\right)^{2} \mathrm{~d} t, L_{2}=\int_{0}^{T} \sum_{i=1}^{N} \sum_{\boldsymbol{j}_{\boldsymbol{i}^{\prime} \in \mathbb{N}_{*}^{N-1}}}\left(\sum_{j_{i}=1}^{\infty} j_{i} C_{2, j} \mathrm{e}^{\left(1+\mu_{j}^{-}\right)(T-t)}\right)^{2} \mathrm{~d} t
$$

and

$$
\begin{aligned}
L_{3} & =\int_{0}^{T} \sum_{i=1}^{N} \sum_{j_{i^{\prime} \in \mathbb{N}_{*}^{N-1}}}\left(\sum_{j_{i}=1}^{\infty} j_{i}(-1)^{j_{i}} C_{1, j} \mathrm{e}^{\left(1+\mu_{j}^{+}\right)(T-t)}\right)^{2} \mathrm{~d} t, \\
L_{4} & =\int_{0}^{T} \sum_{i=1}^{N} \sum_{j_{i^{\prime} \in \mathbb{N}_{*}^{N-1}}}\left(\sum_{j_{i}=1}^{\infty} j_{i}(-1)^{j_{i}} C_{2, j} \mathrm{e}^{\left(1+\mu_{j}^{-}\right)(T-t)}\right)^{2} \mathrm{~d} t,
\end{aligned}
$$

where, for each $i \in\{1, \ldots, N\}$, we have denoted

$$
{j_{i}}^{\prime}:=\left(j_{1}, \ldots, j_{i-1}, j_{i+1}, \ldots, j_{N}\right) \in \mathbf{N}_{*}^{N-1} .
$$

Let us start by taking

$$
C_{1, j}=C_{2, j}=0 \text { if } \exists i \in\{1, \ldots, N\} \text { such that } j_{i} \text { is odd. }
$$

Recall that $\boldsymbol{j}=\left(j_{1}, \ldots, j_{N}\right)$. This readily implies that $L_{1}=L_{3}$ and $L_{2}=L_{4}$.

Let now $M>0$ be a sufficiently large even number and $p \in \mathbf{N}$ (to be fixed). Then, we take

$$
\beta_{j}=0 \text { if there exists } i \in\{1, \ldots, N\} \text { such that } j_{i} \notin X_{M, p}:=\{M+2 k: k \in \mathbf{N}, 1 \leq k \leq p\} .
$$

Study of the terms $L_{2}=L_{4}$

Let us take a closer look to $L_{2}$. For this, we develop in power series for $|\boldsymbol{j}|$ large enough (recall that $\lambda_{\boldsymbol{j}}=$ $\left.\pi^{2}|\boldsymbol{j}|^{2}\right)$ :

$$
C_{2, \boldsymbol{j}}=\frac{\beta_{\boldsymbol{j}}}{2}\left(1-\left(1-4 / \lambda_{\boldsymbol{j}}\right)^{-1 / 2}\right)=-\frac{\beta_{\boldsymbol{j}}}{2} \sum_{k \geq 1} \frac{(k-1 / 2) !}{k !}\left(\frac{4}{\lambda_{j}}\right)^{k}
$$

and

$$
\begin{aligned}
\mathrm{e}^{\left(1+\mu_{j}^{-}\right)(T-t)} & =\exp \left\{\left(1-\frac{\lambda_{j}}{2}+\frac{\lambda_{j}}{2}\left(1-\sum_{k \geq 1} \frac{(k-3 / 2) !}{2(k !)}\left(\frac{4}{\lambda_{j}}\right)^{k}\right)\right)(T-t)\right\} \\
& =\exp \left\{-(T-t) \sum_{k \geq 2} \frac{(k-3 / 2) !}{k !}\left(\frac{4}{\lambda_{j}}\right)^{k-1}\right\}=1-\frac{T-t}{\lambda_{j}}+O\left(\lambda_{j}^{-2}\right) .
\end{aligned}
$$

From (3.7) and (3.8), the term $C_{2, j} \mathrm{e}^{\left(1+\mu_{j}^{-}\right)(T-t)}$ is given, for $|\boldsymbol{j}|$ large enough, by

$$
C_{2, j} \mathrm{e}^{\left(1+\mu_{j}^{-}\right)(T-t)}=-\beta_{j}\left(\frac{1}{\lambda_{j}}+\frac{3-(T-t)}{\lambda_{j}^{2}}+R_{j}\right)
$$

where $R_{j}=O\left(1 / \lambda_{j}^{3}\right)$. 
Then, let us freeze $i \in\{1, \ldots, N\}$ and impose the following $p^{N-1}$ equations for the unknowns $\beta_{\boldsymbol{j}}$ :

$$
\sum_{j_{i} \in X_{M, p}} j_{i} \frac{\beta_{j}}{\lambda_{j}}=0 \quad \forall \dot{j}^{\prime} \in X_{M, p}^{N-1}
$$

Since this is done for each $i \in\{1, \ldots, N\}$, we have imposed $N p^{N-1}$ equations. The linearity of system (3.10) allows us to choose $\beta_{\boldsymbol{j}}$ such that $\left|\beta_{\boldsymbol{j}}\right| \leq C$ with $C$ independent of $M$.

Thus, coming back to the expression of $L_{2}$, we have

$$
L_{2}=\int_{0}^{T} \sum_{i=1}^{N} \sum_{j_{i^{\prime}} \in X_{M, p}^{N-1}}\left(\sum_{j_{i} \in X_{M, p}} j_{i} \beta_{j} \frac{3-(T-t)}{\lambda_{j}^{2}}+j_{i} \beta_{j} R_{j}\right)^{2} \mathrm{~d} t .
$$

Consequently,

$$
L_{2}+L_{4} \leq \frac{C}{M^{6}}
$$

with $C$ independent of $M$.

Study of the terms $L_{1}=L_{3}$

From the equation of $\mu_{j}^{+}$(see (3.4)), we find

$$
\begin{aligned}
\exp \left\{\left(1+\mu_{\boldsymbol{j}}^{+}\right)(T-t)\right\} & =\exp \left\{-\lambda_{\boldsymbol{j}}(T-t)\right\} \exp \left\{(T-t)\left(1+\sum_{k \geq 1} \frac{(k-3 / 2) !}{k !}\left(\frac{4}{\lambda_{j}}\right)^{k-1}\right)\right\} \\
& :=\exp \left\{\left(2-\lambda_{\boldsymbol{j}}\right)(T-t)\right\} \exp \left\{(T-t) D_{\boldsymbol{j}}\right\},
\end{aligned}
$$

where $D_{\boldsymbol{j}}$ goes to zero (like $|\boldsymbol{j}|^{-2}$ ) as $|\boldsymbol{j}|$ goes to infinity. With this, the expression of $L_{1}$ is given by

$$
\sum_{i=1}^{N} \sum_{\boldsymbol{j}_{i}^{\prime} \in X_{M, p}^{N-1}} \int_{0}^{T} \exp \left\{\left(4-2 N M^{2} \pi^{2}\right)(T-t)\right\} g_{M, i, \boldsymbol{j}}(t) \mathrm{d} t
$$

where, for each $i \in\{1, \ldots, N\}$ and each $\boldsymbol{j}_{i}^{\prime} \in X_{M, p}^{N-1}$, we have denoted $g_{M, i, j}:=\left(f_{M, i, j}\right)^{2}$ with

$$
f_{M, i, \boldsymbol{j}}(t)=\sum_{j_{i} \in X_{M, p}} j_{i} C_{1, \boldsymbol{j}} \exp \left\{\left(-2 \pi^{2}\left(|\boldsymbol{j}|^{2}-N M^{2}\right)+D_{\boldsymbol{j}}\right)(T-t)\right\} .
$$

As in the one dimensional case, for every $i \in\{1, \ldots, N\}$ and every $j_{i}{ }^{\prime} \in X_{M, p}^{N-1}$, we require the functions $g_{M, i, j}$ to satisfy

$$
g_{M, i, j}^{(h)}(T)=0 \quad \forall 0 \leq h \leq 6 .
$$

From the definition of $g_{M, i, j}$, these conditions are satisfied as long as

$$
f_{M, i, j}^{(h)}(T)=0 \quad \forall 0 \leq h \leq 3 .
$$

We have thus imposed $4 N p^{N-1}$ equations here.

Then, integrating by parts in (3.12), we find the following expression for $L_{1}$ :

$$
\sum_{i=1}^{N} \sum_{\boldsymbol{j}_{\boldsymbol{\prime}^{\prime}} \in X_{M, p}^{N-1}}\left(\sum_{h=0}^{6} \frac{\exp \left\{\left(4-2 N M^{2} \pi^{2}\right) T\right\}}{\left(4-2 N M^{2} \pi^{2}\right)^{h+1}} g_{M, i, \boldsymbol{j}}^{(h)}(0)+\int_{0}^{T} \frac{\exp \left\{\left(4-2 N M^{2} \pi^{2}\right)(T-t)\right\}}{\left(4-2 N M^{2} \pi^{2}\right)^{7}} g_{M, i, \boldsymbol{j}}^{(6)}(t) \mathrm{d} t\right) .
$$


Since $\left|g_{M, i, j}^{(6)}\right| \leq C M^{8}$, we have that

$$
\left|L_{1}\right| \leq \frac{C}{M^{6}},
$$

where the constant $C>0$ may depend on $p$ and $N$ but does not depend on $M$.

In these two steps, we have imposed $5 N p^{N-1}$ equations while we have $p^{N}$ unknowns. Then, it suffices to take $p=6 N$ and a choice of non-trivial $\beta_{j}^{\prime} s$ is possible. We call $\varphi^{M}$ the corresponding solution to (3.1), which satisfies

$$
\left\|\mathrm{e}^{T-t} \frac{\partial \varphi^{M}}{\partial n}\right\|_{L^{2}((0, T) \times \partial \Omega)}^{2} \leq \frac{C}{M^{6}} .
$$

One can prove like in dimension one the estimate

$$
\left\|\varphi^{M}(0, \cdot)\right\|_{L^{2}(\Omega)}^{2} \geq \frac{\widehat{C}_{0}}{M^{4}}
$$

with $\widehat{C}_{0}>0$ independent of $M$.

\section{Construction of the initial condition}

Following the same ideas as in the one dimensional case, we construct an initial condition $\hat{y}_{0} \in L^{2}(\Omega)$ such that the null controllability of (2.1) does not hold. First, all the previous estimates hold if we suppose that $M$ is a multiple of $2 p$, since we only used that $M$ is even and large enough. In fact, let us replace $M$ by $2 p M$ and $p$ by $6 N$ in the above computations.

Then, from (3.5) and (3.15) we deduce the existence of $\left(k_{1}, \ldots, k_{N}\right) \in\{1, \ldots, 6 N\}^{N}$ such that for the index

$$
\boldsymbol{j}_{0}(M)=\left(12 N M+2 k_{1}, \ldots, 12 N M+2 k_{N}\right),
$$

we have

$$
\frac{1}{2^{N}}\left(C_{1, j_{0}} \mathrm{e}^{\mu_{j_{0}}^{+} T}+C_{2, j_{0}} \mathrm{e}^{\mu_{j_{0}}^{-} T}\right)^{2} \geq \frac{\widehat{C}_{0}}{(6 N)^{N} M^{4}} .
$$

Then, we define

$$
\hat{y}_{0}=\sum_{\ell \geq 1} \frac{1}{\ell^{3 / 4}} w_{j_{0}(\ell)} \in L^{2}(\Omega)
$$

(recall that $w_{\boldsymbol{j}}$ was defined in (3.2)). Let us prove that for any $v \in L^{2}((0, T) \times \partial \Omega)$, the solution $y$ of

$$
\begin{cases}y_{t}-\Delta y-\int_{0}^{t} \Delta y \mathrm{~d} \tau=0 & \text { in } Q \\ y=v & \text { on }(0, T) \times \partial \Omega \\ y(0, \cdot)=\hat{y}_{0}(\cdot) & \text { in } \Omega\end{cases}
$$

satisfies $y(T, \cdot) \not \equiv 0$. Arguing by contradiction, let $v \in L^{2}((0, T) \times \partial \Omega)$ such that $y(T, x)=0$ for all $x \in \Omega$. Then, by duality with $\varphi^{M}$, we have

$$
-\int_{\Omega} \varphi^{M}(0, x) \hat{y}_{0}(x) \mathrm{d} x+\int_{0}^{T} \int_{\partial \Omega} v(t, x) \frac{\partial \varphi^{M}}{\partial \nu} \mathrm{d} \sigma \mathrm{d} t+\int_{0}^{T} \int_{\partial \Omega}\left(\int_{0}^{t} v(s, x) \mathrm{d} s\right) \frac{\partial \varphi^{M}}{\partial \nu}(t, x) \mathrm{d} \sigma \mathrm{d} t=0 .
$$

From the previous choice of $\hat{y}_{0}$ and (3.16), we have that

$$
\left|\int_{\Omega} \varphi^{M}(0, x) \hat{y}_{0}(x) \mathrm{d} x\right| \geq \frac{C_{1}}{M^{11 / 4}} .
$$


Finally, using (3.14), we obtain

$$
\left|\int_{0}^{T} \int_{\partial \Omega} v(t, x) \frac{\partial \varphi^{M}}{\partial \nu} \mathrm{d} \sigma+\int_{0}^{T} \int_{\partial \Omega}\left(\int_{0}^{t} v(s, x) \mathrm{d} s\right) \frac{\partial \varphi^{M}}{\partial \nu}(t, x) \mathrm{d} \sigma \mathrm{d} t\right| \leq \frac{C}{M^{3}} .
$$

This contradicts identity (3.18) by taking $M$ large enough.

\section{REFERENCES}

[1] A.V. Fursikov and O.Yu. Imanuvilov, Controllability of Evolution Equations, Seoul National University, Korea Lect. Notes. 34 (1996).

[2] O.Yu. Imanuvilov, Controllability of parabolic equations (Russian) Mat. Sb. 186 (1995) 109-132; translation in Sb. Math. 186 (1995) 879-900.

[3] S. Ivanov and L. Pandolfi, Heat equation with memory: Lack of controllability to rest. J. Math. Anal. Appl. 355 (2009) 1-11.

[4] G. Lebeau and L. Robbiano, Contrôle exact de l'équation de la chaleur (French). [Exact control of the heat equation]. Commun. Partial Differ. Equ. 20 (1995) 335-356.

[5] J.-L. Lions, Exact controllability, stabilizability and perturbations for distributed systems. SIAM Rev. 30 (1988) 1-68.

[6] J.-L. Lions and E. Magenes, Non-homogeneous boundary value problems and applications I, Translated from the French by P. Kenneth, edited by Springer-Verlag, New York, Heidelberg. Die Grundlehren der Mathematischen Wissenschaften. 181 (1972).

[7] D.L. Russell, Controllability and stabilizability theory for linear partial differential equations. Recent progress and open questions. SIAM Rev. 20 (1978) 639-739.

[8] R. Temam, Navier-Stokes equations, Theory and numerical analysis, edited by North Holland Publishing Co., Amsterdam, New York, Oxford Studies in Math. Appl. 2 (1977). 